\title{
The Effect of Disorder in an Orbitally Ordered Jahn-Teller Insulator
}

\author{
Sanjeev Kumar ${ }^{1}$, Arno P. Kampf ${ }^{1}$, and Pinaki Majumdar ${ }^{2}$ \\ 1 Institute of Physics, Theoretical Physics III, Center for Electronic Correlations and Magnetism, \\ University of Augsburg, D-86135 Augsburg, Germany \\ 2 Harish-Chandra Research Institute, Chhatnag Road, Jhusi, Allahabad 211 019, India
}

\begin{abstract}
We study a two dimensional, two-band double-exchange model for $e_{g}$ electrons coupled to JahnTeller distortions in the presence of quenched disorder using a recently developed Monte-Carlo technique. In the absence of disorder the half-filled system at low temperatures is an orbitally ordered ferromagnetic insulator with a staggered pattern of Jahn-Teller distortions. We examine the finite temperature transition to the orbitally disordered phase and uncover a qualitative difference between the intermediate and strongly coupled systems, including a thermally driven insulator to metal crossover in the former case. Long range orbital order is suppressed in the presence of disorder and the system displays a tendency towards metastable states consisting of orbitally disordered stripe-like structures enclosing orbitally ordered domains.
\end{abstract}

PACS numbers: 71.10.-w, 75.47.Lx, 81.16.Rf

May 11, 2018

\section{INTRODUCTION}

The undoped perovskite manganites, for example $\mathrm{LaMnO}_{3}$, are orbitally ordered antiferromagnetic insulators at low temperature. The magnetic order is of A-type with ferromagnetic planes coupled antiferromagnetically in the transverse direction. The orbital order is of Ctype, anti-correlated with the magnetic order [1], and is accompanied by long range ordering of the Jahn-Teller (JT) distortions of the $\mathrm{MnO}_{6}$ octahedra. Upon hole doping, e.g. in $\mathrm{La}_{1-x} \mathrm{Ca}_{x} \mathrm{MnO}_{3}$, the system evolves via a ferromagnetic insulating phase towards an orbitally disordered (OD) ferromagnetic metal, which exhibits colossal magnetoresistance (CMR) near the Curie temperature 2]. Quenched disorder has been widely acknowledged as a crucial ingredient for the understanding of hole-doped manganites [3, 4]. It is the source of a variety of phenomena, both in real materials as well as in model calculations, like the coexistence of metallic and insulating phases [5, 6, 7, 8, 9], thermally driven metal to insulator transitions 10, 11, 12, 13, and the suppression of charge order in half-doped systems [14, 15].

Although the 'fame' of the manganites rests on the CMR observed near optimal hole doping $(x \sim 0.3)$ the low doping materials also display a rich variety of phenomena [16, 17]. Hole doping leads to progressive loss of orbital order $(\mathrm{OO})$, a reduction of the transport gap, increasing isotropy in the effective magnetic exchange, and an insulator (I) to metal (M) transition [2, 18, 19]. Beyond transport and thermodynamic indicators, NMR and neutron scattering experiments have revealed an inhomogeneous - possibly phase separated - state in the low doping regime [20, 21]. The interplay of doping, disorder, and thermal fluctuations thus presents an intriguing problem even in the insulators. In typical hole doped manganite compounds such as $\mathrm{RE}_{1-x} \mathrm{AE}_{x} \mathrm{MnO}_{3}$, where $\mathrm{RE}(\mathrm{AE})$ denotes a trivalent (divalent) rare-earth (alkaline-earth) ion, the disorder arises from the difference in the ionic radii of the RE and $\mathrm{AE}$ ions and hence, is tied to a variation in the hole concentration, vanishing for the stoichiometric compositions at $x=0$ and $x=1$. Conceptually, however, it is useful to disentangle the effects of increasing hole density and increasing disorder on the OO-OD and IM transitions. In that spirit, this paper focuses on the effect of disorder and varying electronlattice coupling at $x=0$ in a two-orbital model for the Mn $e_{g}$-electrons coupled to JT lattice distortions and to the $t_{2 g}$-derived core spins. The combined effect of disorder and hole doping has been briefly reported elsewhere [9]. A model system, which includes disorder remaining at $x=0$ is experimentally relevant for systems with isoelectronic substitutions such as $\mathrm{RE}_{1-y} \mathrm{RE}_{y}^{\prime} \mathrm{MnO}_{3}$ [22].

The manganites are complex materials involving the the interplay of spin, orbital, lattice, and charge degrees of freedom. In the cubic perovskite structure, the 5 -fold degeneracy of the Mn- $d$ levels is lifted by crystal fields leading to two manifolds of three $t_{2 g}$ and two $e_{g}$ orbitals. The energetically low-lying $t_{2 g}$ levels contain three electrons and give rise to a well localized $S=3 / 2$ spin. The $e_{g}$ electrons are itinerant and interact with the distortions of the $\mathrm{MnO}_{6}$ octahedra via the JT coupling and with the core spins via Hund's rule coupling [16]. The full complexity of these interactions is hard to handle in a realistic two or three dimensional situation. Let us quickly survey the attempts to understand the $x=0$ state before describing our approach.

Different points of view have been reported regarding the relative importance of the inter- and intra- orbital Hubbard interactions and the JT interaction in the manganites [23, 24]. Although the electron-electron (ee) interactions are large in these systems, it has been suggested that the parent insulator can not be considered as a canonical Mott insulator 25]. Surprisingly, the orbitally ordered insulating groundstate at $x=0$ can be understood from a variety of starting points. For strong Hubbard interactions a spin-orbital t-J model has been derived and studied within mean-field approximation [26, 27] leading to an orbitally ordered ground- 
state. The orbitally ordered groundstate also emerges from Hartree-Fock band-structure calculations considering the Mn-3d and O-2p orbitals [28, 29]. Monte-Carlo studies of models including JT coupling and ignoring the local Coulomb repulsion also explain the OO-I state and tend to describe the system as a JT insulator [30]. In fact e-e and JT interactions are known to have qualitatively similar consequences and instead of competing they reenforce each other 31. More recently it was shown that a Fermi surface nesting instability at weak coupling can also be the source for an orbitally ordered groundstate in the presence of Hubbard interactions [32]. It is also known that a more realistic modelling of the lattice taking into acount the individual oxygen displacements of $\mathrm{MnO}_{6}$ octahedra and their cooperative effects, can by itself lead to a staggered JT distorted, and hence orbitally ordered, groundstate [33]. Retaining all the complications of the real materials is a difficult task, particularly when we wish to advance beyond mean field theory. The principal purpose of the present study instead, is to clarify the influence of thermal fluctuations and disorder on the orbitally ordered insulating groundstate. Here we ignore the e-e interactions and the cooperative character of the lattice distortions. The similar effects of e-e and JT interactions in determining the nature of the groundstate suggests that the effect of disorder may also be similar for the two cases.

The remainder of the paper is organized as follows. In Section II we specify the model and briefly describe our Monte-Carlo simulation technique. The results are presented in Sec. III, starting with the non-interacting system and subsequently introducing the JT coupling and quenched disorder. The non disordered zero temperature limit is independently analyzed by using variational calculations, while the clean strong coupling limit studied via an effective classical model.

\section{MODEL AND METHOD}

We consider a two-band model for itinerant $e_{g}$ electrons on a square lattice. The electrons are coupled to JT lattice distortions, $t_{2 g}$ derived $S=3 / 2$ core spins and quenched disorder as described by the Hamiltonian:

$$
\begin{aligned}
H= & \sum_{\langle i j\rangle \sigma}^{\alpha \beta} t_{\alpha \beta}^{i j}\left(c_{i \alpha \sigma}^{\dagger} c_{j \beta \sigma}+\text { h.c. }\right)+\sum_{i}\left(\epsilon_{i}-\mu\right) n_{i} \\
& -J_{H} \sum_{i} \mathbf{S}_{i} . \boldsymbol{\sigma}_{i}-\lambda \sum_{i} \mathbf{Q}_{i} \cdot \boldsymbol{\tau}_{i}+\frac{K}{2} \sum_{i} \mathbf{Q}_{i}^{2} .
\end{aligned}
$$

Here, $c$ and $c^{\dagger}$ are annihilation and creation operators for $e_{g}$ electrons and $\alpha, \beta$ are summed over the two Mn$e_{g}$ orbitals $d_{x^{2}-y^{2}}$ and $d_{3 z^{2}-r^{2}}$, which are labelled $(a)$ and $(b)$ in what follows. $t_{\alpha \beta}^{i j}$ denote the hopping amplitudes between $e_{g}$ orbitals on nearest-neighbor sites and have the cubic perovskite specific form: $t_{a a}^{x}=t_{a a}^{y} \equiv t$, $t_{b b}^{x}=t_{b b}^{y} \equiv t / 3, t_{a b}^{x}=t_{b a}^{x} \equiv-t / \sqrt{3}, t_{a b}^{y}=t_{b a}^{y} \equiv t / \sqrt{3}$, where $x$ and $y$ mark the spatial directions [30]. Disorder is modelled by random on-site potentials $\epsilon_{i}$ with equally probable values $\pm \Delta$, which couples to the local electronic density $n_{i}$. The $e_{g}$-electron spin is locally coupled to the $t_{2 g}$ spin $\mathbf{S}_{i}$ via the Hund's rule coupling $J_{H}$. The electronic spin is given by $\sigma_{i}^{\mu}=\sum_{\sigma \sigma^{\prime}}^{\alpha} c_{i \alpha \sigma}^{\dagger} \Gamma_{\sigma \sigma^{\prime}}^{\mu} c_{i \alpha \sigma^{\prime}}$, where $\Gamma^{\mu}$ are the Pauli matrices. $\lambda$ denotes the strength of the JT coupling between the distortion $\mathbf{Q}_{i}=\left(Q_{i x}, Q_{i z}\right)$ and the orbital pseudospin $\tau_{i}^{\mu}=\sum_{\sigma}^{\alpha \beta} c_{i \alpha \sigma}^{\dagger} \Gamma_{\alpha \beta}^{\mu} c_{i \beta \sigma} . K$ is a measure of the lattice stiffness, and $\mu$ is the chemical potential. We set $t=1=K$ as our reference energy scale. The JT distortions and the $t_{2 g}$ derived core spins are treated as classical variables, and we set $|\mathbf{S}|=1$. The present study is restricted to half-filling and we explore the variation in the parameters $\lambda$ and $\Delta$ in addition to the temperature $T$.

For further simplification we adopt the limit $J_{H} / t \rightarrow$ $\infty$, which is justified and frequently used in the context of manganites [7, 11]. In this limit the electronic spin at site $i$ is tied to the orientation of the core spin $\mathbf{S}_{i}$. Transforming the fermionic operators to this local spin reference frame leads to the following 'spinless' model for the $e_{g}$ electrons:

$$
\begin{gathered}
H=\sum_{\langle i j\rangle}^{\alpha \beta}\left(\tilde{t}_{\alpha \beta}^{i j} c_{i \alpha}^{\dagger} c_{j \beta}+\text { h.c. }\right)+\sum_{i}\left(\epsilon_{i}-\mu\right) n_{i} \\
-\lambda \sum_{i} \mathbf{Q}_{i} \cdot \boldsymbol{\tau}_{i}+\frac{K}{2} \sum_{i} \mathbf{Q}_{i}^{2} .
\end{gathered}
$$

The new hopping amplitudes have an additional dependence on the core spin configurations and are given by:

$$
\frac{\tilde{t}_{\alpha \beta}}{t_{\alpha \beta}}=\cos \frac{\theta_{i}}{2} \cos \frac{\theta_{j}}{2}+\sin \frac{\theta_{i}}{2} \sin \frac{\theta_{j}}{2} e^{-\mathrm{i}\left(\phi_{i}-\phi_{j}\right)} .
$$

Here, $\theta_{i}$ and $\phi_{i}$ denote polar and azimuthal angles for the spin $\mathbf{S}_{i}$. From now on the operator $c_{i \alpha}\left(c_{i \alpha}^{\dagger}\right)$ is associated with annihilating (creating) an electron at site $i$, in the orbital $\alpha$ with spin parallel to $\mathbf{S}_{i}$.

The model given by Eq. (2) is bilinear in the electronic operators and does not encounter the problem of exponentially growing Hilbert space, since all many-particle states can be constructed from Slater determinants of the single-particle states. The difficulty, however, arises from the large phase space in the classical variables $\mathbf{Q}$ and $\mathbf{S}$. At zero temperature the problem reduces to finding the spin and lattice configurations $\left\{\mathbf{S}_{i}, \mathbf{Q}_{i}\right\}$ that minimize the total energy. The energies for a limited number of periodic structures in $\mathbf{Q}$ and $\mathbf{S}$ can be compared analytically. This method is not assured, though, to lead to the true groundstate and often requires additional physics insight for identifying periodic structures, which are prime candidates for the groundstate. The finite temperature properties are not accessible in this manner, since they necessarily require the electronic energies and wavefunc- 
tions in non-periodic structures for the $\mathbf{Q}$ and $\mathbf{S}$ variables. Further complications arise in the presence of disorder and even the groundstate may not belong to the subspace of periodic configurations of the spin and lattice variables. Two controlled methods are available at finite $T$ : (a) dynamical mean-field theory (DMFT) correctly captures the strong coupling physics [34, 35] but is unable to handle spatially correlated inhomogeneous states, which may emerge in the presence of quenched disorder, while, $(b)$ exact diagonalization based Monte Carlo (ED-MC) calculations [16] can handle both thermal fluctuations and disorder, but they are severely size limited.

Here, we will use a scheme which is closely related to ED-MC and employ the travelling cluster approximation (TCA) 36 in order to overcome the small size limitations. This approximation is based on the observation, that the effect of a local update on spin and lattice variables does not 'propagate' long distances via the electrons, and the energy change involved in such a move can be computed by constructing a cluster Hamiltonian around the reference site. This drastically reduces the computational cost and allows access to lattice sizes of $\sim 1000$ sites. For the electronic properties in the thermally equilibrated system we use ED for the full system. The details of this computational scheme have been discussed previously 36]. While TCA forms the backbone of the present study and is used to map out the phase diagram of our model Hamiltonian in the parameter space of $\lambda, \Delta$, and $T$ with detailed real-space information, we also analyze the limiting cases using variational calculations, or an effective classical model for the JT lattice distortions. Apart from providing a comparison to the TCA results, this also enables us to establish a more transparent physical picture of the numerical results.

\section{RESULTS}

\section{A. Orbital order in the groundstate}

We begin by describing the simplest limit of the Hamiltonian in Eq. (2), which is the non-interacting electron system in the absence of disorder. Since the magnetism is purely double-exchange driven, leading to a ferromagnetic (FM) groundstate, a fully polarized core spin state is used for the calculation of the electronic dispersion relation. For $\lambda=0$ the spectrum is straightforwardly obtained by Fourier transformation to momentum space:

$$
c_{i \alpha}^{\dagger}=\frac{1}{N} \sum_{\mathbf{k}} e^{i \mathbf{k} \cdot \mathbf{r}_{\mathbf{i}}} d_{\mathbf{k} \alpha}^{\dagger} .
$$

This gives

$$
H=\sum_{\mathbf{k}, \alpha \beta} \epsilon_{\alpha \beta}(\mathbf{k}) d_{\mathbf{k} \alpha}^{\dagger} d_{\mathbf{k} \beta}
$$

with $\epsilon_{\alpha \beta}(\mathbf{k})=-2 t_{\alpha \beta}^{x} \cos \left(k_{x}\right)-2 t_{\alpha \beta}^{y} \cos \left(k_{y}\right)$. Diagonalizing this Hamiltonian we obtain
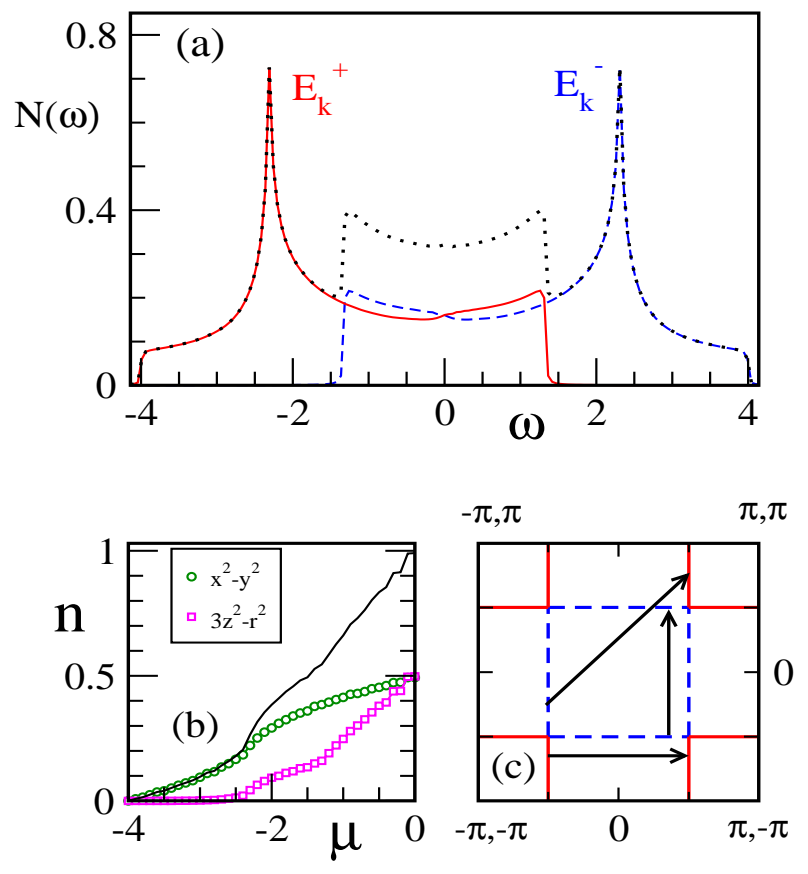

FIG. 1: (Color online) (a) Density of states for the noninteracting Hamiltonian. Solid (dashed) line is for the $E_{\mathbf{k}}^{+}$ $\left(E_{\mathbf{k}}^{-}\right)$band and the total density of states is shown as the dotted line. (b) Filling of the $d_{x^{2}-y^{2}}$ and $d_{3 z^{2}-r^{2}}$ orbitals as a function of the chemical potential. The solid line is the total filling $n$. (c) Fermi surfaces for the non-interacting model at $n=1$. The solid (dashed) segment of the Fermi surface arises from the band $E_{\mathbf{k}}^{+}\left(E_{\mathbf{k}}^{-}\right)$. The three nesting wave-vectors are indicated by the arrows.

$$
E_{\mathbf{k}}^{ \pm}=\frac{\epsilon^{+}(\mathbf{k})}{2} \pm \sqrt{\left(\frac{\epsilon^{-}(\mathbf{k})}{2}\right)^{2}+\epsilon_{a b}^{2}(\mathbf{k})}
$$

where $\epsilon^{ \pm}(\mathbf{k})=\epsilon_{a a}(\mathbf{k}) \pm \epsilon_{b b}(\mathbf{k})$.

Fig. 1(a) shows the band-resolved and the total density of states (DOS) corresponding to the dispersion given in Eq. (6). The electronic DOS is given by:

$$
N(\omega)=\frac{1}{N} \sum_{n} \delta\left(\omega-E_{n}\right)
$$

where $E_{n}$ denotes the eigenvalues of the Hamiltonian. For the band-resolved DOS we use $E_{\mathbf{k}}^{+}$or $E_{\mathbf{k}}^{-}$in place of $E_{n}$. Fig. 1(b) plots the filling of the $d_{x^{2}-y^{2}}$ and $d_{3 z^{2}-r^{2}}$ orbitals as a function of the chemical potential. These are simply the groundstate expectation values of the number operators $d_{\mathbf{k} a}^{\dagger} d_{\mathbf{k} a}$ and $d_{\mathbf{k} b}^{\dagger} d_{\mathbf{k} b}$.

While the occupancy of the two orbitals is equal at $n=1, d_{x^{2}-y^{2}}$ is preferred for $0<n<1$. Fig. 1(c) shows the Fermi surface at electron filling $n=1$, which corresponds to $\mu=0$. From panel (a) it is clear that both bands are partially filled at $\mu=0$ and the Fermi surface consists of contributions from both bands. While each of 
the bands $E_{\mathbf{k}}^{ \pm}$provides a segment of the Fermi surface, nested with wavevectors $(\pi, 0)$ and $(0, \pi)$, there is also interband nesting with wavevector $(\pi, \pi)$. The existence of the nesting wavevectors and a therefore a divergent non-interacting susceptibility induces an ordering instability in the presence of interactions. For example, the inclusion of an infinitesimal electron-lattice coupling, $\lambda$, is expected to lead to a lowering in the electronic energy by stabilizing the lattice patterns, which are compatible with a selected nesting wavevector. Similar arguments have been invoked earlier for the same band dispersion to show that the presence of weak e-e interactions leads to orbital ordering at half filling [32].

In order to confirm this in the context of electronlattice interactions, we perform a variational calculation to search for the lowest energy state within a restricted set of possible groundstates. As discussed earlier, the $T=0$ problem amounts to finding the spin and lattice configurations $\left\{\mathbf{S}_{i}, \mathbf{Q}_{i}\right\}$, which minimize the total energy. Since the groundstate is known to be ferromagnetic, we are left with the problem of determining the minimumenergy lattice configuration $\{\mathbf{Q}\}$ only, for which we set up a restricted variational calculation. We consider variational states of the type $\mathbf{Q}_{i}=\mathbf{Q} e^{\mathbf{i} \mathbf{q} \cdot \mathbf{r}_{i}}$ and compare energies for $\mathbf{q}=(0,0),(0, \pi),(\pi, 0)$ and $(\pi, \pi)$. The variational parameters are the magnitude of the distortion $Q$ and the orientation of $\mathbf{Q}$ in the $Q_{x}-Q_{z}$ plane parameterized by an angle $\zeta$, with $\tan (\zeta)=Q_{x} / Q_{z}$. For this restricted set of lattice configurations the Hamiltonian matrix is reduced to a $2 \times 2$ or $4 \times 4$ form, which is diagonalized to evaluate the total energy and to construct an approximate $T=0$ phase diagram. The matrix for the uniform distortions $(\mathbf{q}=\mathbf{0})$ is

$$
\left(\begin{array}{cc}
\epsilon_{11}(\mathbf{k})-\lambda Q_{z} & \epsilon_{12}(\mathbf{k})-\lambda Q_{x} \\
\epsilon_{12}(\mathbf{k})-\lambda Q_{x} & \epsilon_{22}(\mathbf{k})+\lambda Q_{z}
\end{array}\right)
$$

and the matrix for $\mathbf{q}=(0, \pi),(\pi, 0)$ or $(\pi, \pi)$ is

$$
\left(\begin{array}{cccc}
\epsilon_{11}(\mathbf{k}) & \epsilon_{12}(\mathbf{k}) & -\lambda Q_{z} & -\lambda Q_{x} \\
\epsilon_{12}(\mathbf{k}) & \epsilon_{22}(\mathbf{k}) & -\lambda Q_{x} & \lambda Q_{z} \\
-\lambda Q_{z} & -\lambda Q_{x} & \epsilon_{11}(k+\mathbf{q}) & \epsilon_{12}(\mathbf{k}+\mathbf{q}) \\
-\lambda Q_{x} & \lambda Q_{z} & \epsilon_{12}(\mathbf{k}+\mathbf{q}) & \epsilon_{22}(\mathbf{k}+\mathbf{q})
\end{array}\right)
$$

The results of the variational calculation are summarized in Fig. 2. A staggered pattern of JT distortions with an arbitrary orientation angle $\zeta$ is schematically shown in Fig. 2(a). The minimum energy is obtained for $\zeta_{A}=\pi / 2$ and $\zeta_{B}=3 \pi / 2$. The origin of purely $Q_{x^{-}}$ type distortion patterns is tied to the specific structure of the hopping parameters $t_{\alpha \beta}$ and especially to the sign difference between $t_{a b}^{x}$ and $t_{a b}^{y}$. For $t_{\alpha \beta}^{x} \equiv t_{\alpha \beta}^{y}$ the minimum energy configuration corresponds to a staggered distortion pattern with $\zeta_{A}=\tan ^{-1}\left[2 t_{a b} /\left(t_{a a}-t_{a b}\right)\right]$ and $\zeta_{B}=\pi+\zeta_{A}$. For $t_{a b}^{x}=t_{a b}^{y}=t / \sqrt{3}$ this leads to $\zeta_{A}=\pi / 3$ and $\zeta_{B}=4 \pi / 3$, and for $t_{a b}^{x}=t_{a b}^{y}=-t / \sqrt{3}$ the corresponding angles are $2 \pi / 3$ and $5 \pi / 3$. The relative sign difference between $t_{a b}^{x}$ and $t_{a b}^{y}$ in cubic perovskites, thus leads to a conflict regarding the orientation angles for (a)
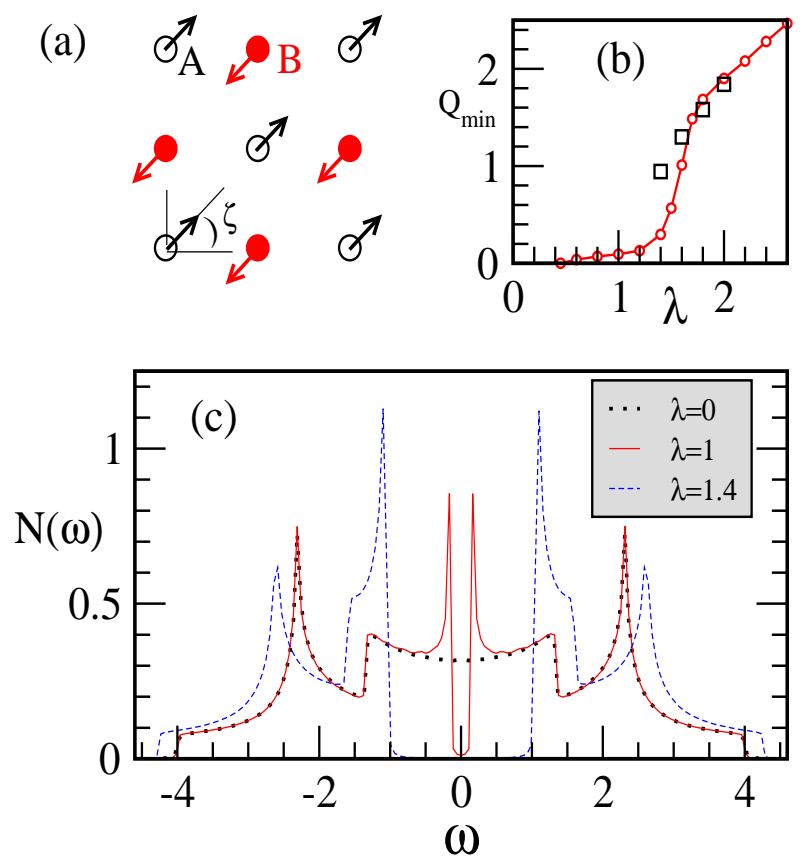

FIG. 2: (Color online) (a) Schematic picture of a staggered distortion pattern for the JT distortion vectors $\mathbf{Q}$. The arrows indicate the directions of $\mathbf{Q}$, with an arbitrary angle $\zeta$. The groundstate corresponds to $\zeta_{A}=\pi / 2$, and $\zeta_{B}=3 \pi / 2$. (b) The variation of the magnitude of the distortion shown in (a) with $\lambda$. The circles are obtained from the variational calculation and the squares are from the Monte-Carlo simulations. (c) Density of states for $\lambda=0,1$ and 1.4 in the variational groundstate. Lattice sizes of up to $2000 \times 2000$ sites are used in the variational study.

the directions of $\mathbf{Q}$ on the two sublattices and $\zeta_{A}=\pi / 2$, $\zeta_{B}=3 \pi / 2$ result as a compromise. The $Q_{x}$-type distortion patterns can also be motivated from the structure of the effective classical model at strong JT coupling, which is again related to the perovskite specific hopping parameters. Since the lattice distortions are coupled to the orbital pseudospin, $Q_{x}$-type ordering in the JT distortions is accompanied by $\tau_{x}$-type staggered order in the orbital sector.

Fig. 2(b) shows the the magnitude $Q_{\min }$ of the distortion corresponding to the minimum energy state as a function of the electron-lattice coupling. For weak $\lambda$ there is a 'BCS' like instability leading to an exponentially small $Q_{\min }$ and a correspondingly small gap in the DOS 37]. The window $0.0<\lambda<1$ roughly corresponds to this weak coupling regime and displays a slow rise in $Q_{\min }$ with $\lambda$. For $1<\lambda<2$, the intermediate coupling regime, $Q_{\min }$ increases rapidly crossing over to the strong coupling asymptote, $Q_{\min } \propto \lambda$, for $\lambda>2$. The squares mark the data points obtained from TCA, which are discussed later. Fig. 2(c) shows the DOS for the lattice structure of panel (a) in the weak and intermediate coupling regime.

At strong coupling it is reasonable to start from the 
atomic limit and to assume that the electrons are sitelocalized by strong lattice distortions [38]. In that case the total energy per site, which includes the electronic and the elastic energy, is given by

$$
E_{t o t}=-\lambda|\mathbf{Q}|+\frac{K}{2}|\mathbf{Q}|^{2} .
$$

Minimizing the total energy to obtain the optimum distortion $Q_{\min }$, one finds $Q_{\min }=\lambda / K$. This is precisely the observed behavior for $\lambda>2$. Such a self-trapped object is usually referred to as a polaron and the associated energy is termed single-polaron energy $E_{p}=\lambda^{2} / 2 K$. In this paper, a 'polaron' always refers to a 'static polaron' since the lattice is treated in the adiabatic limit. The qualitative differences between intermediate and strong coupling will become apparent in the finite temperature studies using TCA.

\section{B. Monte Carlo results}

\section{TCA in the clean limit}

The finite $T$ problem is solved using the TCA method described earlier. In this case an unrestricted search is performed with respect to the spin and lattice configurations and the temperature is reduced step by step to obtain the groundstate. Fig. 3(a) shows the temperature dependence of the $\mathbf{q}=(\pi, \pi) \equiv \mathbf{q}_{0}$ component of the lattice structure factor,

$$
D_{Q}(\mathbf{q})=N^{-2} \sum_{i j}\left\langle\mathbf{Q}_{i} \cdot \mathbf{Q}_{j}\right\rangle_{t h} e^{-\mathrm{iq} \cdot\left(\mathbf{r}_{i}-\mathbf{r}_{j}\right)} .
$$

Here and below $\langle\ldots\rangle_{t h}$ denotes the average over thermal equilibrium configurations. All the TCA results are obtained on a $24 \times 24$ lattice and a $4 \times 4$ travelling cluster. $D_{Q}\left(\mathbf{q}_{0}\right)$ is a measure of the staggered ordering tendency of the JT distortions. Since the lattice distortions are coupled to the orbital pseudospin the same tendency is transferred to the analogously defined orbital structure factor $D_{\tau}\left(\mathbf{q}_{0}\right)$. Therefore $D_{Q}\left(\mathbf{q}_{0}\right)$ serves as an indicator for the staggered ordering of both, the lattice distortions and the orbital pseudospin. The point of inflection in the temperature dependence of $D_{Q}\left(\mathbf{q}_{0}\right)$ is taken as the orbital ordering transition temperature $T_{O O}$. Fig. $3(\mathrm{~b})$ shows the variation of $T_{O O}$ with $\lambda$. The symbols are TCA results and the solid line is a guide to eye. The extrapolation of the solid line to $\lambda<0.8$ is based on the existence of a BCS-like instability at weak $\lambda$ due to Fermi-surface nesting as already encountered in the variational calculations. In the weak-coupling regime $T_{O O}$ is proportional to the energy gap $\Delta_{G}$ in the DOS. The dashed line indicates the result of a strong coupling expansion, which leads to the proportionality $T_{O O} \propto t^{2} / E_{p}$. The details of the strong coupling expansion are discussed in the appendix. The non-monotonic behavior of $T_{O O}$ is thus naturally understood by merging the weak and strong coupling limits. The experimental results on $\mathrm{REMnO}_{3}$ for
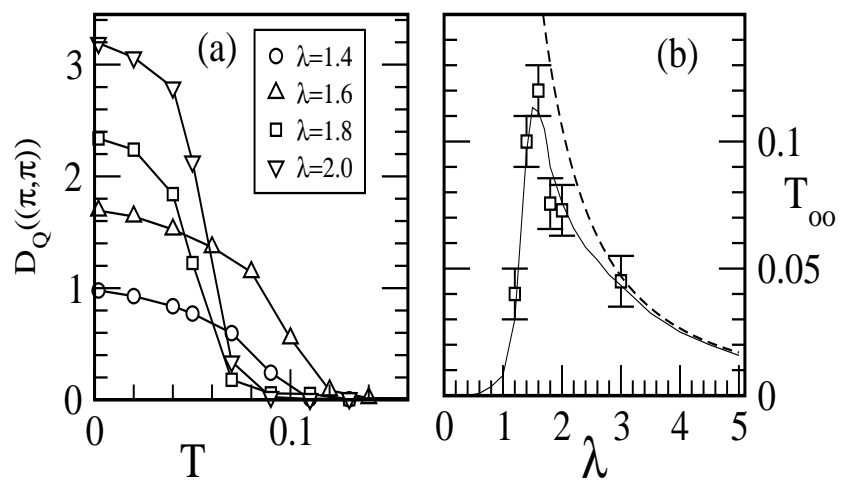

FIG. 3: (a) Temperature dependence of the lattice structure factor $D_{Q}((\pi, \pi))$. (b) Variation of the orbital-ordering transition temperatures $T_{O O}$ with $\lambda$. The squares are the TCA results and the solid line is a guide to eye. The strong coupling asymptotic form is also shown by the dashed line.

the accessible range of decrease in mean ionic radii show an increase in $T_{O O}$ [39]. A reduction in ionic radii leads to a decrease in the bandwidth, which translates to an effective increase in $\lambda / t$ in our model. This suggests that the relevant regime for the strength of JT coupling should be $\lambda<1.8$. A more direct estimate for the value of $\lambda$ is obtained by comparing the ratio $\Delta_{G} / T_{O O}$ between our results and the experiments. For $\mathrm{LaMnO}_{3}, \Delta_{G} \sim 0.5 \mathrm{eV}$ [18] and $T_{O O} \sim 700 K$ [39] leading to $\Delta_{G} / T_{O O} \sim 8$. This value is approximately reproduced for $\lambda \sim 1.2$. In real materials additional interactions may lead to a renormalization of the effective JT coupling 31], therefore these numbers should be considered only as a rough estimate.

Since the orbital order is tied to the ordering of the JT distortions, it is useful to track the temperature evolution of the distribution function for the lattice distortions

$$
P(Q)=\frac{1}{N}\left\langle\sum_{i} \delta\left(Q-\left|\mathbf{Q}_{i}\right|\right)\right\rangle_{t h},
$$

where $\left|\mathbf{Q}_{i}\right|$ denotes the magnitude of the local lattice distortions for an equilibrium configuration. The temperature dependence of $P(Q)$ is shown in Figs. 4(a) and 4(b) for moderate and strong JT coupling strengths, respectively. The low $T$ distributions are peaked at $Q=Q_{\text {min }}$, suggesting an unimodular structure at $T=0$ and supporting the variational results for the groundstate. The symbols represent the TCA data and the solid lines follow from simple arguments for the expected structure of the distribution function $P(Q)$ : The single-site energy for an electron self-trapped by a distortion of magnitude $Q$ is given by $E=-\lambda Q+K / 2 Q^{2}$. The probability that this site has a distortion with magnitude $Q$ at a given temperature $T$ is then given by $P(Q) \propto \exp \left[-\left(E-E_{0}\right) / T\right]$, where $E_{0}$ is the groundstate energy correspnding to $Q=$ $Q_{\text {min }}$. This leads to $P(Q) \propto \exp \left[-\left(Q-Q_{\text {min }}\right)^{2} / 2 T\right]$, assuming that the functional form of the energy does not change for finite $T$. Using the $Q_{\min }$ data of the 

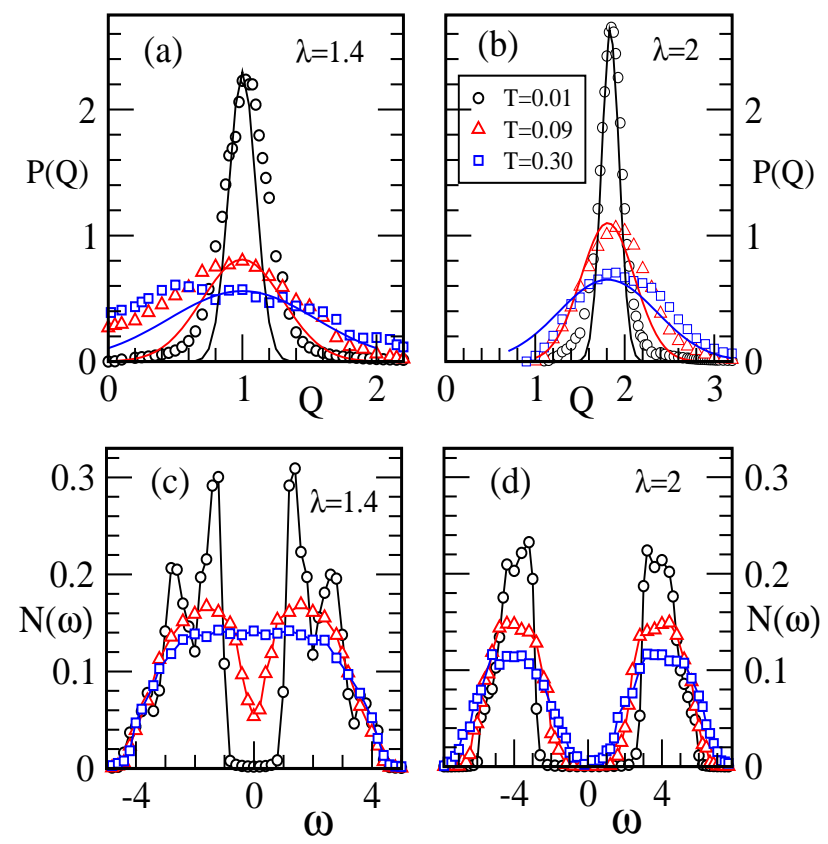

FIG. 4: (Color online) Temperature evolution of the distribution function for the magnitude of the lattice distortions $P(Q)$ for moderate (a), and strong (b) electron-lattice coupling strengths. Symbols are the Monte-Carlo data and the solid lines correspond to the naive expectation based on the atomic limit. The density of states for the parameters corresponding to (a) and (b) is shown in (c) and (d), respectively.

TCA results these functions are plotted as solid lines in Figs. 4(a) and 4(b). The TCA results show that, $P(Q=0)$ is finite for $T \geq T_{O O}$ at $\lambda=1.4$; thus some of the electrons can apparently escape from JT-distorted cages upon heating. This suggests the possibility of an insulator to metal crossover and will be discussed in the next section. The naive analysis described above does not apply for weak coupling, as is apparent from the large deviations of the firm lines from the symbols, infact, even the width at low temperature of the distribution function is not captured by the corresponding Gaussian function. For $\lambda=2$ however, the electrons are well trapped by the self-generated JT distortions even at $T \sim 3 T_{O O}$. The Gaussian functions with width $\propto \sqrt{T}$ are reasonable fits to the TCA data, implying that $\lambda=2$ is close to the regime where single-site analysis is valid. This clarifies a crucial difference between the weak and the strong coupling systems despite the fact that the orbital order in the groundstate is the same in both limits. In the strong coupling regime the distortions exist even at high temperatures and the orbital ordering transition corresponds to the alignment of the distortion vectors at $T \sim T_{O O}$. This is analogous to the ordering in a spin model with magnetic moments of fixed magnitude. The mechanism for orbital ordering at weak to moderate coupling relies on a simultaneous generation and ordering of the lattice distortions.

Although the stability of these results has been checked for system sizes ranging from $N=8^{2}$ to $N=32^{2}$, a detailed finite size scaling has not been performed. The observed qualitative difference between the moderate and strong coupling systems raise the possibility, that the orbital order to disorder transitions in the two cases may be qualitatively different. Experimentally it is known that the transition in $\mathrm{LaMnO}_{3}$ is first order in nature and becomes second order for $\mathrm{PrMnO}_{3}$ and $\mathrm{NdMnO}_{3}$, which are smaller bandwidth materials [22, 40, 41]. Although the origin for a first order nature of the transition in $\mathrm{LaMnO}_{3}$ is suggested to be anharmonic coupling between JT distortions and volume strain [42], the bandwidth variation may also be playing a role. Figs. 4(c) and 4(d) show the electronic DOS for the same parameter values as used in (a) and (b), respectively. The low $T$ DOS is gapped, consistent with our variational results. The gap fills up for $T \sim T_{O O}$ for weaker $\lambda$ but persists to much larger $T$ for strong coupling. The origin of the gap in the DOS is therefore very different for the two cases and finds supports in the structure in $P(Q)$. Indeed, if the distortions are well formed even at large $T$, as is the case for strong coupling, the DOS is gapped due to 'self-trapping'. On the other hand a gap in the DOS for moderate coupling is tied to the existence of orbital order, which in turn requires a long range ordered pattern for the lattice distortions. Note that the sizes of both $Q_{\min }$ and the gap in the DOS obtained in the TCA calculation match very well with the variational calculations (see Fig. 2(b)).

\section{The effect of disorder}

Although the presence of disorder is usually tied to the hole-doped materials, it can also be realized in compositions like $\mathrm{RE}_{1-y} \mathrm{RE}_{y}^{\prime} \mathrm{MnO}_{3}$. Moreover it is useful to study the effect of disorder without involving the complications of finite hole density. Therefore, given the presence of orbital order in the half-filled clean system, we now include the effects of quenched disorder. Although the Monte-Carlo results provide us with the full finite- $T$ information, we first present our results on the disorder effects at low $T$. In this section we focus on the thermodynamic quantities, which are averaged over $\sim 10$ realizations of quenched disorder. Fig. 5(a) shows the staggered component of the lattice structure factor at low $T$ as a function of the disorder strength $\Delta$. The critical value $\Delta_{c}$ for the disappearence of orbital order in the groundstate is estimated from these data and further confirmed by the $T$ dependence of $D_{Q}\left(\mathbf{q}_{0}\right) . D_{Q}\left(\mathbf{q}_{0}\right) \sim O(1)$ is considered to indicate orbital order and $D_{Q}\left(\mathbf{q}_{0}\right) \sim O\left(1 / N^{2}\right)$ implies an orbitally disordered phase. A non-monotonic dependence of $\Delta_{c}$ on the JT coupling strength is observed as shown in Fig. 5(b). The similarity between $\Delta_{c}(\lambda)$ and $T_{O O}(\lambda)$ (see Fig. 3(b)) suggests that the effects of quenched disorder and thermal fluctuations are similar in weakening the long range orbital order.

For further details on the disorder driven orbital order to disorder crossover, we show the disorder evolution of 


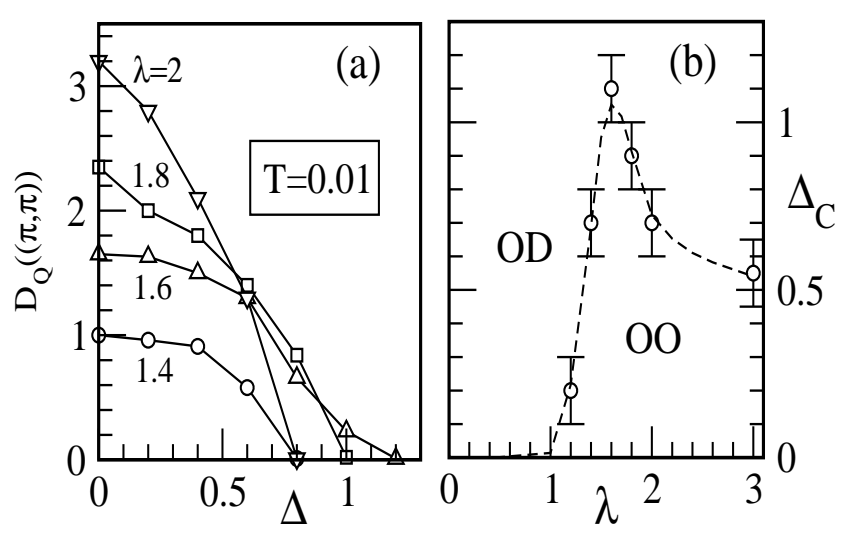

FIG. 5: (a) Lattice structure factor $D_{Q}((\pi, \pi))$ at $T=0.01$ as a function of the disorder strength $\Delta$. (b) Critical disorder strength $\Delta_{c}$ required to spoil the orbital order in the groundstate as a function of $\lambda$.

the distribution function $P(Q)$ for the JT distortions for two representative values of $\lambda$ in Fig. 6(a) and 6(b). These results are very similar to the $T$ dependence of the distribution function (see Figs. 4(a)-(b)). Interestingly disorder acts as a delocalizing agent for the weak coupling system, leading to a fraction of sites with very weak JT distortions, hence delocalizing a fraction of electrons from the self generated JT traps. The effect of the disorder on $P(Q)$ is barely visible at large $\lambda$ and $P(Q)$ is not affected in crossing over from the orbitally ordered to the orbitally disordered state. Figs. 6(c) and 6(d) show the effect of disorder on the DOS for the same values of parameters as used in panels (a) and (b). Our earlier suggestion that the gap in the DOS is related to the orbital ordering for weak electron-lattice coupling is confirmed. For $\Delta=$ 0.8 a pseudogap feature replaces the clean gap in the DOS. The strong coupling DOS is only slightly affected by disorder and in particular the gap survives even in the orbitally disordered phase. This is again similar to the temperature dependence of the DOS for the weak and strong coupling limits.

The effect of disorder is also different for the finite $T$ properties between weak and strong coupling. Fig. 7 shows the temperature dependence of $D_{Q}\left(\mathbf{q}_{0}\right)$ for various $\Delta$ at moderate (panel (a)) and strong (panel (b)) JT couplings. The $T=0$ value of $D_{Q}\left(\mathbf{q}_{0}\right)$ reduces with increasing disorder in both cases. The detailed real-space analysis of this reduction will be presented later, but it is worth mentioning already here that orbitally disordered stripe-like structures emerge in the disordered system at low temperatures, specially in the strong coupling regime. While the inclusion of disorder affects the saturation values for $D_{Q}\left(\mathbf{q}_{0}\right)$ in a similar manner for both $\lambda=1.4$ and $\lambda=2.0, T_{O O}$ scales show an interesting contrast between the two coupling strengths. $T_{O O}$ reduces gradually for $\lambda=1.4$ with increasing disorder strength and eventually both the saturation value of $D_{Q}\left(\mathbf{q}_{0}\right)$ and $T_{O O}$ approach zero as the long range orbital order in the groundstate is lost. On the other hand, $T_{O O}$ does not
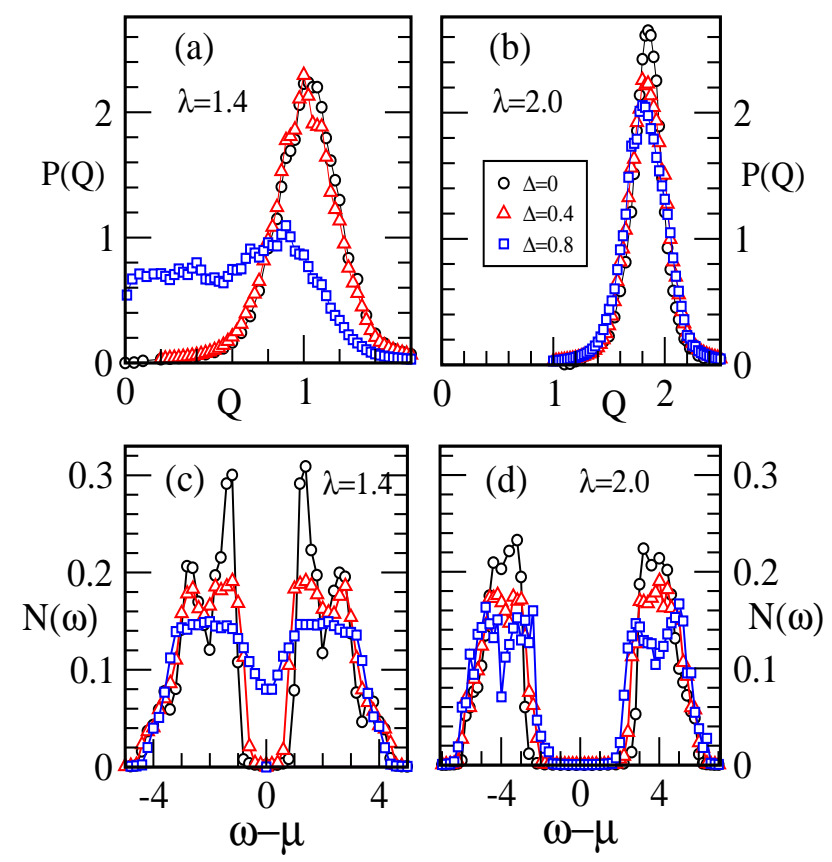

FIG. 6: (Color online) Disorder dependence of the distribution function $P(Q)$ for moderate (a), and strong (b) electronlattice coupling strengths. The density of states for the parameters corresponding to (a) and (b) is shown in (c) and (d), respectively.

change with disorder for $\lambda=2.0$ and drops abruptly to zero close to $\Delta_{c}$, when the system crosses over to the orbitally disordered phase.

For further confirmation on the difference between the weak and strong coupling systems we compute the optical conductivity $\sigma(\omega)$ using the Kubo formula with the exact eigenstates [43]. The resistivity $\rho$ is approximated by the inverse of $\sigma\left(\omega_{\min }\right)$, where $\omega_{\min }=20 t / N \sim 0.03 t$ is the lowest reliable frequency scale for $\sigma(\omega)$ calculations on our $N=24^{2}$ system. Fig. $7($ c) shows the resistivity for $\lambda=1.4$ as a function of $\lambda$ for different values of disorder strength. A sharp upturn in the resistivity is observed upon cooling, which is clearly due to the onset of orbital ordering. For all values of $\Delta<\Delta_{c}$ a change of slope in the resistivity is observed, indicating a thermally driven I-M crossover. For $\Delta \geq \Delta_{c}$, the system retains $d \rho / d T>0$ for all $T$, indicating a disorder-induced metallization of the insulator. While the Anderson localization effects may not allow a metallic state in lower dimensions, the I-M transition induced by quenched disorder via the destruction of orbital order is expected in three dimensional systems too. The observation of a disorder-induced metallic state is not new and has been reported before in the context of charge ordering [14, 15. The temperature dependence of the resistivity for $\lambda=2$ is shown in Fig. 7(d). This strong coupling system displays a more robust insulating behavior. The effect of thermal fluctuations and/or disorder is barely visible on the resistivity. Contrary to the weak-coupling system, the onset of or- 

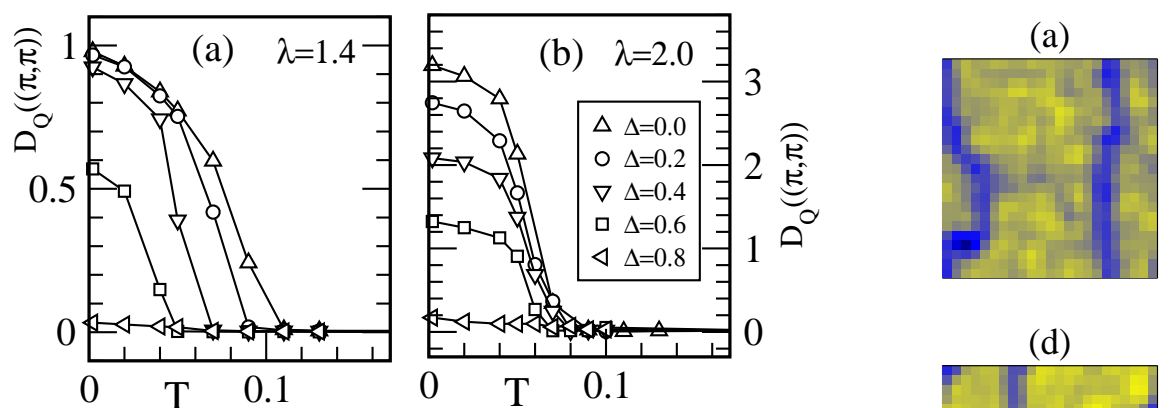

(d)
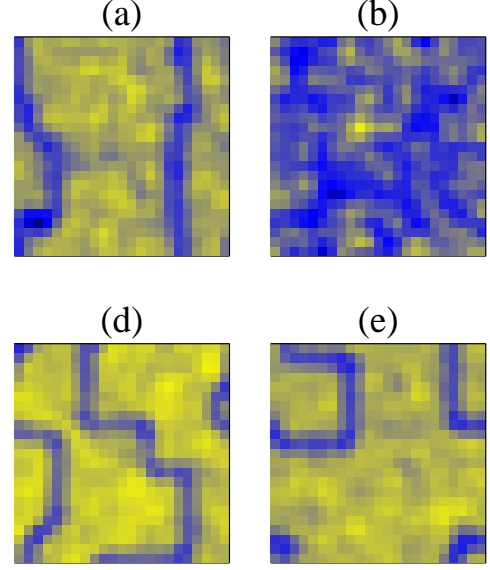

(e)
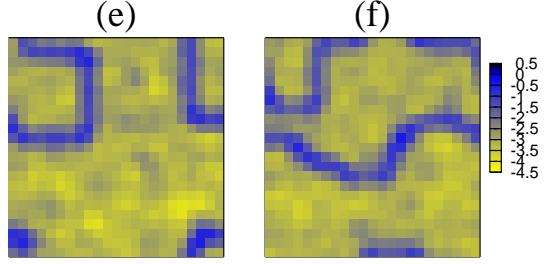

(c)

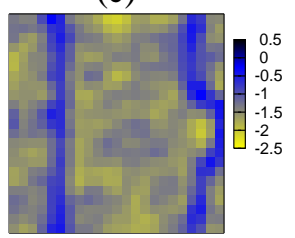

(f)
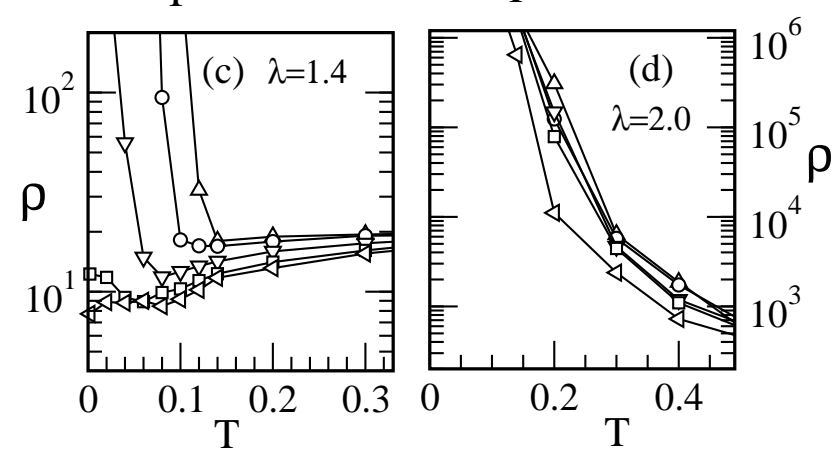

FIG. 7: Temperature dependence of the lattice structure factor $D_{Q}((\pi, \pi))$ for varying disorder strengths at (a) $\lambda=1.4$ and (b) $\lambda=2.0$. Resistivity $\rho$ in units of $\hbar / \pi e^{2}$, as a function of temperature for (c) $\lambda=1.4$ and (d) $\lambda=2.0$ for the same values of $\Delta$ as in (a) and (b).

bital ordering is not reflected in $\rho(T)$.

We observe a monotonic decrease of $T_{O O}$ scales upon increasing disorder in the experimentally relevant regime of moderate JT coupling. In real systems however, it is difficult to isolate the individual effect of quenched disorder. Isoelectronic rare-earth substitution series such as in $\mathrm{La}_{1-y} \mathrm{Nd}_{y} \mathrm{MnO}_{3}$, involve a simultaneous change of effective bandwidth and disorder 22. The increase in $T_{O O}$ upon increasing $y$ in this series is understood mainly from the increase in the average ionic radius but changes in $y$ are necessarily accompanied by variations in disorder.

One of the advantages of the Monte-Carlo method is the access to detailed real-space information. This is especially useful for determining the mechanism leading to the loss of orbital order upon increasing disorder strength. In Fig. 8 we show the local spatial correlations of the JT distortions, as represented by the quantity $C_{Q}^{i}=\frac{1}{4} \sum_{\delta} \mathbf{Q}_{i} \cdot \mathbf{Q}_{i+\delta}$, where $\delta$ is summed over the nearest neighbors of site $i$. Therefore $C_{Q}^{i} \sim-Q_{\min }^{2}$ represents a region of staggered orbital ordering while $C_{Q}^{i} \sim 0$ corresponds to an OD neighborhood. Panels (a)-(c) show the groundstate pattern for $C_{Q}^{i}$ at moderate strength of the JT coupling for three typical disorder realizations. In some cases orbitally disordered filamentary structures are observed. These OD domain walls separate the orbitally ordered regions, and a $\pi$ phase shift appears in crossing from one OO region to the other. The patterns for $C_{Q}^{i}$ at strong coupling are shown in panels (d)-(f). Again the data are presented

FIG. 8: (Color online) Local spatial correlations $C_{Q}^{i}$ (see text) of the Jahn-Teller distortions at $T=0.01$ for three typical realizations of quenched disorder. Panels (a)-(c) correspond to $\lambda=1.6, \Delta=0.5$, and panels (d)-(f) are for $\lambda=2.0$, $\Delta=0.6$.

for three typical realizations of quenched disorder. The tendency to form filamentary structures of orbitally disordered regions is even stronger for strong JT coupling. A careful analysis of these structures shows that these are not 'unique' groundstates of the system and should be understood as metastable states. Even for a fixed realization of disorder a different starting configuration of the lattice variables in the Monte-Carlo simulations leads to a different filamentary structure. Moreover, in most cases these states are higher in energy as compared to the states with no domain walls. Upon doping the OO insulator, similar stripe-like structures of OD regions appear as true groundstates and serve as the prefered locations for the doped holes [9]. While the earlier study involved a combined effect of quenched disorder and hole-doping, here we propose that disorder itself leads to the existence of metastable low energy configurations with filamentary OD regions enclosing $\mathrm{OO}$ domains. The disorder in our model couples to the total charge density and in order to understand how the effect is transferred to the orbital degrees of freedom, we analyze the effect of single impurity in the orbitally ordered groundstate. Considering a repulsive impurity, the impurity site leads to a slightly lower value of charge density. Since the band dispersion is such that $n_{a} \neq n_{b}$ for $n \neq 1$, the sites with $n<1$ has a finite value of $\tau^{z}$. These finite $\tau^{z}$ values upset the purely $\tau^{x}$-type order.

\section{CONCLUSIONS}

In summary, we have studied a two-band doubleexchange model at half filling for $e_{g}$ electrons coupled to Jahn-Teller distortions in the presence of quenched disorder in two dimensions. The existence of long range orbital order and the accompanying staggered order in the 
Jahn-Teller distortions in the groundstate is verified by means of variational calculations, a strong coupling analysis, and real-space Monte-Carlo simulations. The qualitative difference between the moderate and strong JahnTeller coupling systems is reflected in the nature of the orbital ordering transitions in the two regimes. A thermally driven crossover from an insulator to a metal is observed for weak to moderate coupling, in contrast to a robust insulating phase at strong coupling. Quenched disorder destablizes the orbitally ordered groundstate and leads to the appearance of metastabe states with orbitally disordered domain walls separating the orbitally ordered regions. These metastable, inhomogeneous states in the insulating half-filled system are important for the understanding of structures and phase transitions in the hole doped systems $[9]$.

\section{ACKNOWLEDGMENTS}

SK and APK gratefully acknowledge support by the Deutsche Forschungsgemeinschaft through SFB 484. PM was supported during part of this work by the EPSRC (UK) and Trinity College at Cambridge University, by the Royal Society (UK) at Oxford University, and by the Institut Laue-Langevin at Grenoble. Simulations were performed on the Beowulf Cluster at HRI.

\section{APPENDIX: EFFECTIVE CLASSICAL MODEL}

In the limit of large JT coupling, the electrons are essentially site-localized and the atomic limit $\left(t_{\alpha \beta} \equiv 0\right)$ is a good starting point. The electronic eigenvalues for a single-site problem are given by $\pm \lambda|\mathbf{Q}|$. Only the lower electronic level is occupied for the half-filled case. Minimizing the sum of electronic and lattice energies for the optimum value of the magnitude of the JT distortion $\mathbf{Q}$ leads to $Q_{\min }=\lambda / K$. While the magnitude of the JT distortion is determined locally by a balance between the elastic and the electronic energy, there is no prefered direction for the orientation of $\mathbf{Q}$. This degeneracy is lifted by including a finite hopping amplitude for electrons and leads to an effective classical model for the orientations of the JT lattice vectors $\mathbf{Q}$. The derivation is straightforward and the main steps are outlined below:

Consider a two site problem with $H^{\prime}=H_{0}+V$

$$
\begin{aligned}
H_{0} & =-\lambda \sum_{i=1,2}\left\{Q_{i x} \tau_{i}^{x}+Q_{i z} \tau_{i}^{z}\right\}+\frac{K}{2}\left|\mathbf{Q}_{i}\right|^{2} \\
V & =\sum_{\alpha \beta}\left(t_{\alpha \beta} c_{1 \alpha}^{\dagger} c_{2 \beta}+\text { h.c. }\right)
\end{aligned}
$$

The eigenvalues of $H_{0}$ are $\pm \lambda\left|\mathbf{Q}_{i}\right|$ with $i=1,2$. The eigenvectors $\left|\psi_{ \pm}\right\rangle$are site-localized, and consist of linear combinations of the two orbitals at a site. The eigenvector for the eigenvalue $-\lambda\left|\mathbf{Q}_{i}\right|\left(\lambda\left|\mathbf{Q}_{i}\right|\right)$ corresponds to $\boldsymbol{\tau}$ pointing parallel (antiparallel) to $\mathbf{Q}$, which has the full

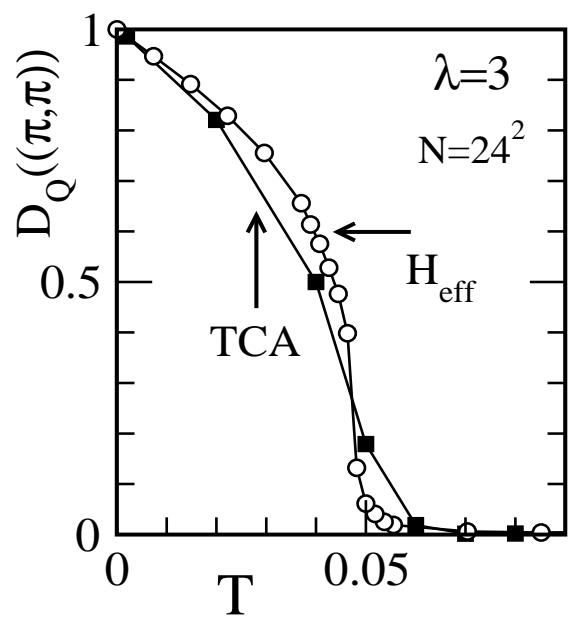

FIG. 9: Lattice structure factor $D_{Q}((\pi, \pi))$ as a function of temperature for the effective strong-coupling model compared with the TCA results at $\lambda=3.0 . D_{Q}((\pi, \pi))$ is normalized to its saturation value for the TCA data.

rotational symmetry of the plane. Explicitly, the eigenvectors are:

$$
\begin{aligned}
& \left|\psi_{-}\right\rangle_{i}=\left\{\cos \left(\frac{\zeta_{i}}{2}\right) c_{i a}^{\dagger}+\sin \left(\frac{\zeta_{i}}{2}\right) c_{i b}^{\dagger}\right\}|0\rangle, \\
& \left|\psi_{+}\right\rangle_{i}=\left\{-\sin \left(\frac{\zeta_{i}}{2}\right) c_{i a}^{\dagger}+\cos \left(\frac{\zeta_{i}}{2}\right) c_{i b}^{\dagger}\right\}|0\rangle,
\end{aligned}
$$

where $\zeta_{i}=\tan ^{-1}\left(Q_{i x} / Q_{i z}\right)$ denotes the orientation of the JT distortion vector $\mathbf{Q}_{i}$ in the $Q_{x}-Q_{z}$ plane.

The total energy of this 2 -site system is

$$
E=-\lambda \sum_{i=1,2}\left|\mathbf{Q}_{i}\right|+\frac{K}{2}|\mathbf{Q}|_{i}^{2}
$$

which is minimized by $\left|\mathbf{Q}_{i}\right|=\lambda / K$ independent of $i$. The two electron groundstate of $H_{0}$ thus corresponds to having one electron at each of the two sites with a lattice distortion $|\mathbf{Q}|=\lambda / K$, total energy $E_{0}=-\frac{\lambda^{2}}{K}$ and the groundstate wavefunction $\left|\psi_{0}\right\rangle=\left|\psi_{-}\right\rangle_{1} \otimes\left|\psi_{-}\right\rangle_{2}$.

Turning on the hopping part of the Hamiltonian $V$, the perturbative correction to the energy is given by

$$
\Delta E=\sum_{m} \frac{\left|\left\langle\psi_{m}\left|t_{\alpha \beta}\left(c_{1 \alpha}^{\dagger} c_{2 \beta}+h . c .\right)\right| \psi_{0}\right\rangle\right|^{2}}{E_{0}-E_{m}}
$$

where, $\left|\psi_{m}\right\rangle$ denotes an excited state of $H_{0}$ in the twoelectron subspace. Explicit evaluation leads to

$$
\begin{aligned}
\Delta E= & J_{x} \sin \zeta_{1} \sin \zeta_{2}+J_{z} \cos \zeta_{1} \cos \zeta_{2} \\
& +J_{m}\left(\sin \zeta_{1} \cos \zeta_{2}+\cos \zeta_{1} \sin \zeta_{2}\right),
\end{aligned}
$$

with coupling constants $J_{x}=\frac{K}{\lambda^{2}}\left(t_{a a} t_{b b}+t_{a b}^{2}\right)$, $J_{z}=\frac{K}{\lambda^{2}}\left(\frac{\left(t_{a a}^{2}+t_{b b}^{2}\right)}{2}-t_{a b}^{2}\right)$, and $J_{m}=\frac{K}{\lambda^{2}}\left[t_{a b}\left(t_{a a}-t_{b b}\right)\right]$. 
This energy acts as an effective classical Hamiltonian for the ordering of the distortion vectors $\mathbf{Q}$.

For a comparison with the results obtained from the TCA, we show the temperature dependence of $D_{Q}\left(\mathbf{q}_{0}\right)$ for the effective model and TCA in Fig. 9. These results are obtained on the same lattice sizes as used for the TCA calculations, i.e. $24 \times 24$. The TCA curve is normalized by its $T=0$ value. The coefficients used in $H_{\text {eff }}$ have an additional factor of $1 / 2$ because the system is still paramagnetic when the orbital ordering takes place and the hopping amplitude $t$ has to be replaced by $t \sqrt{\left(1+\mathbf{S}_{i} \cdot \mathbf{S}_{j}\right) / 2}$, which becomes $1 / \sqrt{2}$ in the paramagnetic phase. The $T_{O O}$ scales obtained from $H_{\text {eff }}$ match very well with the TCA scales for $\lambda \geq 3$ and begin to deviate significantly only for $\lambda \leq 2$ (see Fig. 3(b)). The purely $Q_{x}$-type staggered ordering in the JT distortion vectors is also observed within the effective classical Hamiltonian. This can be easily understood by looking at the values of coupling constants $J_{x}, J_{z}$ and $J_{m}$. Again the difference in sign between the values for $J_{m}^{x}$ and $J_{m}^{y}$, which originates from the sign difference between $t_{a b}^{x}$ and $t_{a b}^{y}$, turns out to be crucial.
[1] G. Biotteau, M. Hennion, F. Moussa, J. RodriguezCarvajal, L. Pinsard, A. Revcolevschi, Y. M. Mukovskii, and D. Shulyatev, Phys. Rev. B 64, 104421 (2001).

[2] B. B. Van Aken, O. D. Jurchescu, A. Meetsma, Y. Tomioka, Y. Tokura, and T. T. M. Palstra, Phys. Rev. Lett. 90, 066403 (2003).

[3] L. M. Rodriguez-Martinez and J. P. Attfield, Phys. Rev. B 58, 2426 (1998); ibid. 54, R15622 (1996); ibid. 63, 024424 (2001).

[4] Y. Tomioka and Y. Tokura, Phys. Rev. B 70, 014432 (2004).

[5] M. Uehara, S. Mori, C. H. Chen, and S.-W. Cheong, Nature (London) 399, 560 (1999).

[6] M. Fath, S. Freisem, A. A. Menovsky, Y. Tomioka, J. Aarts, and J. A. Mydosh, Science 285, 1540 (1999).

[7] A. Moreo, M. Mayr, A. Feiguin, S. Yunoki, and E. Dagotto, Phys. Rev. Lett. 84, 5568 (2000).

[8] S. Kumar and P. Majumdar, Phys. Rev. Lett. 92, 126602 (2004).

[9] S. Kumar, A.P. Kampf, and P. Majumdar, preprint cond-mat 0607642

[10] D. Akahoshi, M. Uchida, Y. Tomioka, T. Arima, Y. Matsui, and Y. Tokura, Phys. Rev. Lett. 90, 177203 (2003).

[11] J. A. Vergés, V. Martín-Mayor, and L. Brey, Phys. Rev. Lett. 88, 136401 (2002).

[12] S. Kumar and P. Majumdar, Phys. Rev. Lett. 96, 016602 (2006).

[13] C. Sen, G. Alvarez, H. Aliaga, and E. Dagotto, Phys. Rev. B 73, 224441 (2006).

[14] Y. Motome, N. Furukawa, and N. Nagaosa, Phys. Rev. Lett. 91, 167204 (2003).

[15] G. Alvarez, H. Aliaga, C. Sen, and E. Dagotto, Phys. Rev. B 73, 224426 (2006).

[16] For overviews see, Nanoscale Phase Separation and Colossal Magnetoresistance by E. Dagotto, SpringerVerlag, Berlin 2002 and [17].

[17] Colossal Magnetoresistive Oxides, edited by T. Chatterji, Kluwer Academic Publishers, 2004.

[18] T. T. M. Palstra, A. P. Ramirez, S-W. Cheong, B. R. Zegarski, P. Schiffer, and J. Zaanen, Phys. Rev. B 56, 5104 (1997).

[19] M. Hennion, F. Moussa, P. Lehouelleur, F. Wang, A. Ivanov, Y. M. Mukovskii, and D. Shulyatev, Phys. Rev. Lett. 94, 057006 (2005).

[20] G. Papavassiliou, M. Pissas, M. Belesi, M. Fardis, J. Dolinsek, C. Dimitropoulos, and J. P. Ansermet, Phys. Rev. Lett. 91, 147205 (2003).
[21] M. Hennion, F. Moussa, G. Biotteau, J. RodrguezCarvajal, L. Pinsard, and A. Revcolevschi, Phys. Rev. Lett. 81, 1957 (1998).

[22] D. Bhattacharya, P. Sujatha Devi, and H. S. Maiti, Phys. Rev. B 70, 184415 (2004).

[23] S. Okamoto, S. Ishihara, and S. Maekawa, Phys. Rev. B 65, 144403 (2002).

[24] A. J. Millis, R. Mueller, and B. I. Shraiman, Phys. Rev. B 54, 5405 (1996).

[25] A. Yamasaki, M. Feldbacher, Y.-F. Yang, O. K. Andersen, and K. Held, Phys. Rev. Lett. 96, 166401 (2006).

[26] K. I. Kugel and D. I. Khomskii, Zh. Eksp. Teor. Fiz. 64, 1429 (1973).

[27] S. Ishihara, J. Inoue, and S. Maekawa, Phys. Rev. B 55, 8280 (1997).

[28] T. Mizokawa and A. Fujimori, Phys. Rev. B 51, 12880 (1995).

[29] T. Mizokawa and A. Fujimori, Phys. Rev. B 54, 5368 (1996)

[30] T. Hotta, S. Yunoki, M. Mayr, and E. Dagotto, Phys. Rev. B 60, R15009 (1999).

[31] P. Benedetti and R. Zeyher, Phys. Rev. B 59, 9923 (1999).

[32] D. V. Efremov and D. I. Khomskii, Phys. Rev. B 72, 012402 (2005).

[33] K. H. Ahn, T. Lookman, A. Saxena, and A. R. Bishop, Phys. Rev. B 71, 212102 (2005).

[34] K. Held and D. Vollhardt, Phys. Rev. Lett. 84, 5168 (2000).

[35] A. J. Millis, R. Mueller, and B. I. Shraiman, Phys. Rev. B 54, 5389 (1996).

[36] S. Kumar and P. Majumdar, Eur. Phys. J. B 50, 571 (2006).

[37] S. Blawid and A. J. Millis, Phys. Rev. B 62, 2424 (2000).

[38] A. J. Millis, Phys. Rev. B 53, 8434 (1996).

[39] T. Kimura, S. Ishihara, H. Shintani, T. Arima, K. T. Takahashi, K. Ishizaka, and Y. Tokura, Phys. Rev. B 68, R060403 (2003).

[40] J.-S. Zhou and J. B. Goodenough, Phys. Rev. B 68, 144406 (2003).

[41] T. Chatterji, F. Fauth, B. Ouladdiaf, P. Mandal, and B. Ghosh, Phys. Rev. B 68, 052406 (2003).

[42] T. Maitra, P. Thalmeier, and T. Chatterji, Phys. Rev. B 69, 132417 (2004).

[43] S. Kumar and P. Majumdar, Eur. Phys. J. B 46, 237 (2005). 